\title{
Developing a univariate approach to phase-I monitoring of fuzzy quality profiles
}

\author{
Kazem Noghondarian and Shahram Ghobadi*
}

Department of Industrial Engineering, Iran University of Science \& Technology, Narmak, Tehran, Iran

\begin{tabular}{|c|c|}
\hline A R T I C L E I N F O & A B S T RACT \\
\hline $\begin{array}{l}\text { Article history: } \\
\text { Received } 24 \text { May } 2012 \\
\text { Received in revised format } \\
\text { 12 June } 2012 \\
\text { Accepted June } 122012 \\
\text { Available online } \\
\text { 14 June } 2012 \\
\text { Keywords: } \\
\text { Fuzzy profile } \\
\text { Fuzzy linear regression } \\
\text { Linear programming } \\
\text { Fuzzy individuals control chart } \\
\text { Fuzzy moving-rang control chart }\end{array}$ & $\begin{array}{l}\text { In many real-world applications, the quality of a process or a particular product can be } \\
\text { characterized by a functional relationship called profile. A profile builds the relationships } \\
\text { between a response quality characteristic and one or more explanatory variables. Monitoring the } \\
\text { quality of a profile is implemented to understand and to verify the stability of this functional } \\
\text { relationship over time. In some real applications, a fuzzy linear regression model can represent } \\
\text { the profile adequately where the response quality characteristic is fuzzy. The purpose of this } \\
\text { paper is to develop an approach for monitoring process/product profiles in fuzzy environment. A } \\
\text { model in fuzzy linear regression is developed to construct the quality profiles by using linear } \\
\text { programming and then fuzzy individuals and moving-range (I-MR) control charts are developed } \\
\text { to monitor both intercept and slope of fuzzy profiles to achieve an in-control process. A case } \\
\text { study in customer satisfaction is presented to show the application of our approach and to express } \\
\text { the sensitivity analysis of parameters for building a fuzzy profile. }\end{array}$ \\
\hline
\end{tabular}

\section{Introduction}

In statistical process control (SPC) applications it is assumed that the quality of a process or product can be suitably characterized by the distribution of a quality characteristic. In numerous practical situations, however, the quality of a process or a product is represented and summarized better by a relationship between a quality response variable and one or more explanatory variables. In the literature, the relationship is referred to as profile. Thus, the monitoring and controlling of these kinds of processes and products will be done by monitoring profiles. In other words, profile monitoring is bringing into play the control charts for cases in which the quality of a process or product can be distinguished by a functional relationship between a response variable and one or more explanatory variables.

The analysis of profiles includes two phases, phase I and phase II, as shown in figure 1. In phase I, we analyze a historical set of fixed number of process samples collected over time. Analyzing is done by

* Corresponding author. Tel: +982177240000

E-mail: ghobadi_sh@iust.ac.ir(S. Ghobadi)

(C) 2012 Growing Science Ltd. All rights reserved.

doi: $10.5267 /$ j.ijiec.2012.05.008 
identifying regression lines for each sample. In phase I, we seek to identify any out-of-control regression lines and then to remove them from the data set in order to estimate strictly the in-control regression parameters for use in phase II online monitoring. The main concern in the analysis of phase II is quickly detecting shifts in the process parameters via online monitoring the process profile.

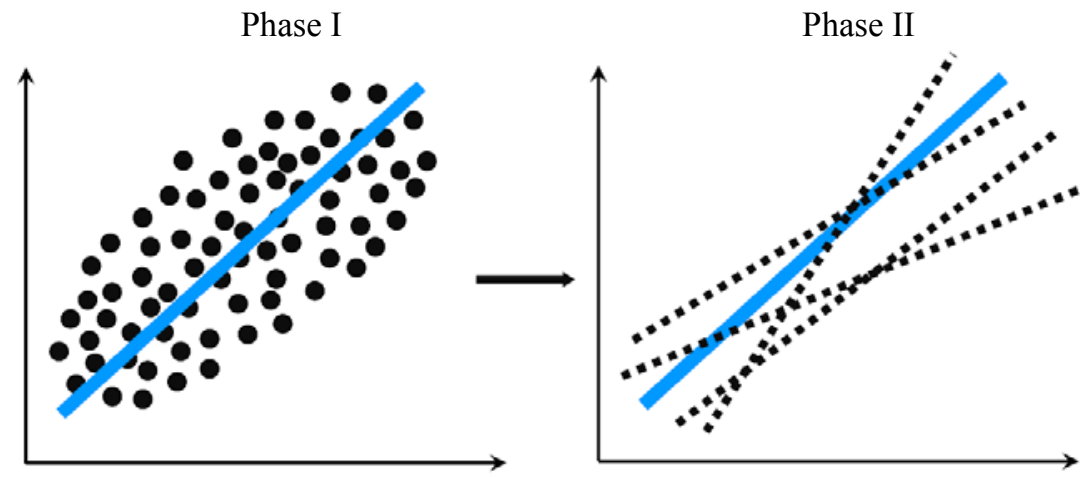

Fig. 1. Phase I \& II in profile monitoring

\section{1. literature review on monitoring the quality profiles in phase I}

Quality profiles can take on several different functional structures such as linear, nonlinear, polynomial, etc, depending on the particular application. In the area of simple linear profile monitoring, Kang and Albin (2000) proposed two methods for monitoring simple linear profiles: bivariate $\mathrm{T}^{2}$ control chart and EWMA/R control charts. Monitoring the relationship between vertical densities of engineered wood boards and depths is considered by Walker and Wright (2002). Kim et al. (2003) coded the $\mathrm{x}$-values to change the average to zero and then applied three separate EWMA control charts. Montgomery (2005) discussed a case where the relationship between the torque and the engine speed can be modeled by a profile. Mahmoud and Woodall (2004) recommended the use of a univariate control chart in conjunction with the F-test to monitor the regression coefficients in phase I. Noorossana et al. (2004) proposed MCUSUM procedure in combination with $\mathrm{R}$ chart proposed by Kang and Albin (2000) to improve the performance of the existing methods in phase II monitoring of simple linear profiles. Zou et al. (2006) and Mahmoud et al. (2007) proposed methods based on likelihood ratio statistics to monitor linear profiles in phase II and I, respectively. Gupta et al. (2006) compared the performance of Kim et al. (2003) method with a method developed by Croarkin and Varner (1982).. Jensen et al. (2008) proposed linear mixed models to account for the autocorrelation within a linear profile. Noorossana et al. (2008) investigated the effect of autocorrelation between linear profiles on the performance of $\mathrm{T}^{2}$ control chart proposed by Kang and Albin (2000). Saghaei et al. (2009) proposed a method based on cumulative sum statistics for monitoring of linear profiles in phase II. Zhang and Wang (2009) constructed control chart based on likelihood ratio for monitoring linear profiles in phase II. Zhu and Lin (2010) proposed some approaches for Monitoring the Slopes of Linear Profiles in both phase I and II. Noorossana et al. (2010) developed four methods based on Principal components analysis for phase I monitoring of multivariate multiple linear regression profiles in a calibration application. Noorossana et al. (2010) proposed the use of three control chart schemes for Phase II monitoring of multivariate simple linear profiles. Chen and Nembhard (2011) developed a high-dimensional control chart for profile monitoring in phase I. The effect of non normality on the performance of control charts for monitoring simple linear profiles is investigated by Noorossana et al. (2011). Eyvazian et al. (2011) proposed four methods based on likelihood ratio approach to monitor multivariate multiple linear profiles in Phase II. Hosseinifard et al. (2011) developed three monitoring methods based on artificial neural networks to monitor linear profiles. Zou et al. (2012) developed a methodology for monitoring general multivariate linear profiles, including the regression coefficients and profile variation in the basis of applying the variable-selection-based multivariate control scheme to the transformations of estimated profile parameters. Amiri et al. (2012) proposed a dimension 
reduction method to overcome the dimensionality problem of some of the methods on phase II monitoring of multiple linear regression profile.

In the previous researches about profile monitoring, all of the studies carried out in the monitoring of profiles deals with crisp quality characteristics in non-fuzzy environment where it is assumed that the process input and output data are not fuzzy. But in many real applications, these data have a kind of uncertainty, fluctuations, impreciseness and vagueness or they may be linguistic attribute data. Information may be incomplete, imprecise, vague, contradictory or deficient in some way or the other, and each one of these various information deficiencies results in different types of uncertainty. Unlike traditional methods in monitoring profiles, fuzzy set approaches can put up with imprecision and uncertainty without loss of performance and effectiveness. Thus, the monitoring of fuzzy profiles can be applied for a wide variety of applications. No work has already been done for monitoring of fuzzy profiles in both phases I and II. In this paper, we introduce some new univariate approaches for monitoring of fuzzy linear profiles in which the relationship between a fuzzy response (fuzzy output) and some explanatory crisp variables (non-fuzzy inputs) is defined by a fuzzy linear regression.

In section 2, we introduce fuzzy linear profile practically. In section 3, we develop a fuzzy regression model to construct fuzzy linear profile for each sample in the basis of linear programming. Fuzzy individuals and moving range (I-MR) control chart are developed in section 4 to monitor the process profiles constructed for each sample. In section 5, we propose developed approaches for detecting outof-control profiles. In section 6, a case study in customer satisfaction is presented to show the application of our approach. Some analysis for fuzzy regression model is done in section 7 to investigate the effect of regression parameters on fuzzy linear profiles.

\section{Fuzzy Linear Profile}

We assume that $m$ random samples from a historical data set are available. For the $j^{\text {th }}$ random sample collected over time, we have the observations $\left(x_{i j}, \tilde{y}_{i j}\right), i=1,2, \ldots, n$, and $j=1,2, \ldots, m$. We assume that the $X$-values are non-fuzzy input and fixed and take the same set of values for each sample. So it means that $x_{i j}=x_{i}$ where $x_{i}$ 's are different levels of a single explanatory crisp variable $\mathrm{X}$. The value $\tilde{y}_{i j}$ is a linguistic quality characteristics and displays a symmetrical triangular fuzzy number with center $y_{i j}$ and width of $s_{i j} \geq 0$ as shown in Fig. 2. For each sample we assume that the linear model relating the independent variable $\mathrm{X}$ to the fuzzy quality response $\mathrm{Y}$ is

$\tilde{Y}_{i j}=\tilde{B}_{0 j}+\tilde{B}_{1 j} X_{i}$,

where regression coefficient $B_{0 j}(r=0 \& 1)$ for sample $\mathrm{j}$ is a symmetrical triangular fuzzy number centered at $b_{r j}$ with width $d_{r j} \geq 0$.
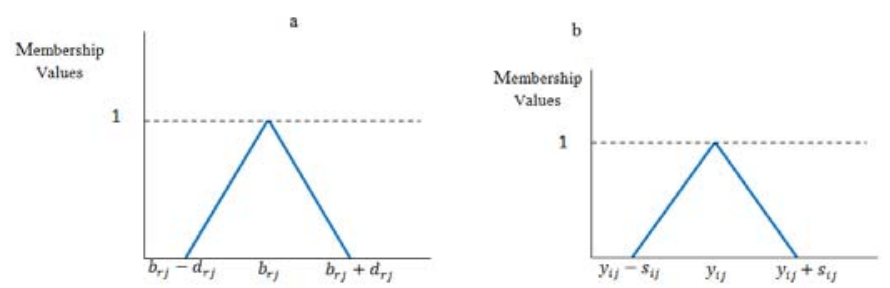

Fig. 2. a. triangular Fuzzy regression coefficients \& b. triangular fuzzy response QC

\section{Constructing Fuzzy Linear Profiles in phase I}

We consider the phase I analysis in which the in-control values of the parameters $\widetilde{B}_{0}$ and $\widetilde{B}_{1}$ in Eq. (1) are unknown. In phase-I analysis we try to find any out-of-control fuzzy regression lines and to remove them from the data set, assuming that the corresponding assignable causes can be identified and removed. This is required to estimate accurately the in-control fuzzy regression parameters for use in 
phase II. If the process is stable and "in-control" then $\tilde{B}_{0 j}=\tilde{B}_{0}$ and $\tilde{B}_{1 j}=\widetilde{B}_{1}$ and all sample data follow underlying model.

$\tilde{Y}=\tilde{B}_{0}+\tilde{B}_{1} X$.

In our proposed approach, we first code the $X$-values so that the average of coded value is zero. This simplifies the resulting analysis and gets rid of much of the need for multivariate monitoring approaches since, in this situation, the least squares estimators of the intercept and slope for each sample are independent fuzzy variables (Kim et al. (2003)).

After transforming the X-values, we obtain an alternative form of the underlying model in Eq. (2) as follows,

$\tilde{Y}=\tilde{A}_{0}+\tilde{A}_{1} X$

where $\tilde{A}_{0}=\tilde{B}_{0}+\tilde{B}_{1} X$ and $\tilde{A}_{1}=\tilde{B}_{1}$ and $X=X_{\text {old }}-\bar{X}_{\text {old }}$. For the $\mathrm{j}^{\text {th }}$ sample, the estimator of $\tilde{A}_{0}$ and $\tilde{A}_{1}$ are $\tilde{A}_{0 j}$ and $\tilde{A}_{1 j}$, respectively. The regression coefficient $\tilde{A}_{r j}$ for sample $j$ is a symmetrical triangular centered at $a_{r j}$ and width $c_{r j} \geq 0$. Both $\tilde{A}_{0 j}$ and $\tilde{A}_{1 j}$ are normally distributed with means $\tilde{A}_{0}$ and $\tilde{A}_{1}$, respectively and they are independent. Thus, we can apply some separate univariate control charts for each sequence of random variables $\tilde{A}_{0 j}$ and $\tilde{A}_{1 j}$ without the problems that would result if the estimators were correlated. In general, there are two main approaches of fuzzy regression models due to different fitting criterion (Tanaka et al. (1989) and Azadeh et al. (2010)). The first approach is based on minimizing the fuzziness of the response variables as an optimal criterion which was first proposed by Tanaka et al. (1989). The advantage of this approach is its simplicity in programming and computation, but it has been criticized to provide too wide ranges in estimation which cannot give much help in the application ((Wang and Tsuar (2000 a,b) and Chang and ayyub(2001) ). The second main approach uses least squares of errors as a fitting criterion to minimize the difference between the estimated and observed responses. Integrating minimum fuzziness criterion and the ordinary least-squares concept into fuzzy regression models is an extension of fuzzy regression and will be developed in this paper to monitor fuzzy profiles. In the proposed approach both objectives are used in the objective function and a multi-objective linear programming is developed.

\subsection{The Developed Model for Constructing $j^{\text {th }}$ Fuzzy Linear profile}

It is assumed that the value $\tilde{y}_{i j}$ is a symmetrical triangular fuzzy number with center $y_{i j}$ and width of $s_{i j} \geq 0$. If we are interested in using part of $\tilde{y}_{i j}$ with minimum confidence level, $h$, where $(0 \leq h \leq 1)$, we should use an interval as follows,

$\left[y_{i j}-(1-h) s_{i j}, y_{i j}+(1-h) s_{i j}\right]$

This interval is the bold boundary lines in figure 3. Here $h$ represents the least confidence level and is called the $h$-cut of the observed values. Similarly, the support of the predicted fuzzy value $\hat{\tilde{y}}_{i j}$ corresponding to a set of values $x_{i}$ with membership at least $h$ is obtained as follows,

$\left[\sum_{r=0}^{1}\left(a_{r j}-(1-h) c_{r j}\right) x_{i}, \quad \sum_{r=0}^{1}\left(a_{r j}+(1-h) c_{r j}\right) x_{i}\right] \quad i=1, \ldots, n$

In previous models used by Ozelkan and Duckstein(2000), Hojati et al.(2004) and Azadeh et al. (2010), fuzzy linear regression has been fitted concerning two objectives: minimizing the width of the regression coefficients and bringing the $h$-cut of the estimated values of response as close as possible to the $h$-cut of the observed values. Each of previous researchers identified the fuzzy regressions models based on one of these two objectives, or considering the other objective as a 
constraint to achieving the objective.

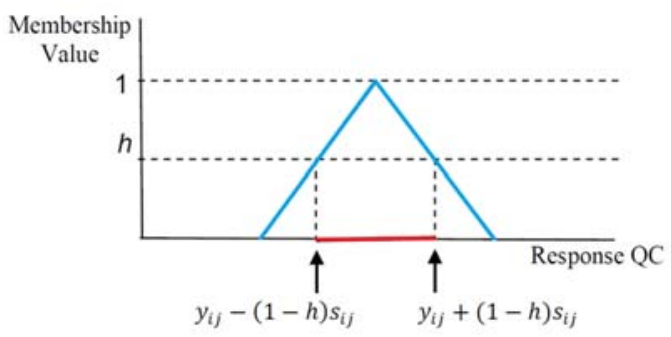

Fig. 3. $h$-cut of each response value in sample j

In our proposed method, both objectives are placed in the objective function and a linear programming is introduced to construct $j^{\text {th }}$ profile as follows.

$\min$

$$
w_{1} \sum_{i=1}^{n} \sum_{r=0}^{1} c_{r}\left|x_{i r}\right|+w_{2} \sum_{i=1}^{n}\left(d_{i U}+d_{i L}\right)
$$

subject to $\sum_{r=0}^{1}\left(a_{r}+(1-h) c_{r}\right) x_{i r} \geq y_{i}+(1-h) s_{i}-d_{i U}$

$$
\begin{aligned}
& \sum_{r=0}^{1}\left(a_{r}-(1-h) c_{r}\right) x_{i r} \leq y_{i}-(1-h) s_{i}+d_{i L} \\
& w_{1}+w_{2}=1 \\
& d_{i L}, d_{i U} \geq 0 \\
& a_{r} \in I R \quad c_{r} \geq 0 \quad r=0,1 \quad i=1, \ldots, n
\end{aligned}
$$

In our model shown in Eq. (6), the first objective minimizes the width of the regression coefficients and so concludes to minimum $h$-cut of the estimated values. The second objective makes the $h$-cut of the estimated values cover the $h$-cut of the observed values. To solve this model, depending on the decision maker's preferences, a weight factor is allocated to each objective. In other word, our developed fuzzy regression model integrates two classic objectives in literature by assigning weight factors to each of them determined by decision maker. Where $w_{1}$ and $w_{2}$ are weight factors determined by the decision maker, and $d_{i U}$ and $d_{i L}$ are the deviation variables and nonnegative.

$$
d_{i U}=\max \left\{0,\left(\max \operatorname{supp} \tilde{y}_{i}-\max \operatorname{supp} \hat{\tilde{y}}_{i}\right)\right\}, d_{i L}=\max \left\{0,\left(\min \operatorname{supp} \hat{\tilde{y}}_{i}-\min \operatorname{supp} \tilde{y}_{i}\right\}\right.
$$

$d_{i U}$ calculates the difference between upper values of the support of $\hat{\tilde{y}}_{i}$ and $\tilde{y}_{i}$ in sample $j$ when the upper value of the support of estimated value $\hat{\tilde{y}}_{i j}$ is smaller than the upper value of the support of observed value $\tilde{y}_{i j}$ in sample $j$. Also, $d_{i L}$ calculates the difference between lower values of the support of the estimated responses $\hat{\tilde{y}}_{i}$ and observed responses $\hat{\tilde{y}}_{i}$ when lower value of the support of $\hat{\tilde{y}}_{i}$ is greater than lower value of the support of $\hat{\tilde{y}}_{i}$ in $j^{\text {th }}$ sample. $w_{1}<w_{2}$ means that the decision maker is risk averser and concluds the $h$-cut of the estimated values envelops the $h$-cut of the related observed values. It means that the second objective is more considerable and of higher weight to him. If the decision maker is risk seeker, he will prefer the $h$-cut of the predicted values to have a narrow width. In this case, the first objective becomes more vital to him and he assigns it a higher weight. Allocating same weights implies the equal importance of both objectives. For each sample we run the above linear program to specify fuzzy linear profile models.

\section{Monitoring of Fuzzy Linear Profiles}

After specifying fuzzy linear profile and estimating related fuzzy coefficients $\tilde{A}_{0 j}$ and $\tilde{A}_{1 j}$ for all samples, we should determine the stability of the process. Several statistical methods have already been developed for monitoring linear profiles in non-fuzzy environment as surveyed in literature review. As 
shown before, nobody developed approaches for monitoring fuzzy quality profiles. In this section we develop some fuzzy controls for monitoring fuzzy linear profiles in phase $\mathrm{I}$ in order to determine the stability of the process and to monitor the estimated fuzzy profile parameters $\tilde{A}_{0 j}$ and $\tilde{A}_{1 j}$ to achieve in-control parameters.

Each profile is composed of two individual fuzzy coefficients $\tilde{A}_{r j}$ which are independent due to coding the explanatory variable $X$ in. Therefore, individual control charts are used for monitoring the mean of each profile parameters, and moving range (MR) control charts are used for monitoring their deviations. Generally in non-fuzzy environment, the data in variable control charts are assumed to be crisp values. But a fuzzy profile is constructed of "uncertain" or "vague" data. The fuzzy set theory is an existing tool for treating the vague data, therefore fuzzy individuals control chart and fuzzy moving range (MR) control chart introduced by Erginel (2008) are developed to monitor the mean and deviation of profile parameters, respectively.

\subsection{Fuzzy Individuals \& Moving Range Control Charts for Intercept $\tilde{A}_{0}$ and Slope $\tilde{A}_{1}$}

In the proposed monitoring approach, the profiles parameters $\tilde{A}_{0}$ and $\tilde{A}_{1}$ are represented by triangular membership functions. The fuzzy center line of individual control chart, $\widetilde{C L}_{\tilde{A}_{r}}$ is the mean of $\mathrm{m}$ fuzzy parameters $\tilde{A}_{r j}$ and is equal to $\overline{\tilde{A}}_{r}=\left(\bar{A}_{r L}, \bar{A}_{r}, \bar{A}_{r U}\right)$ where $\bar{A}_{r L}, \bar{A}_{r}$, and $\bar{A}_{r U}$ are the arithmetic means of $A_{r j L}, A_{r j}$, and $A_{r j U}$, respectively. $A_{r j L}, A_{r j U}$ and $A_{r j}$ are the left, right and middle point of fuzzy regression coefficient $\tilde{A}_{r j}$ as follows,

$A_{r j L}=a_{r j}-c_{r j}$

$A_{r j}=a_{r j}$,

$A_{r j U}=a_{r j}+c_{r j}$.

Also the fuzzy center line of moving range control chart, $\widetilde{C L}_{M R_{r}}$, is the mean of $\mathrm{m}$ parameters $\widetilde{M R}_{r j}$ and it is shown as $\overline{\widetilde{M R}}_{r}=\left(\overline{M R}_{r L}, \overline{M R}_{r}, \overline{M R}_{r U}\right)$ where $\overline{M R}_{r L}, \overline{M R}_{r}$ and $\overline{M R}_{r U}$ are the arithmetic means of $M R_{r j L}, M R_{r j}$ and $M R_{r j U}$ respectively. $\widetilde{M R}_{r j}$ is a triangular fuzzy number and calculates as follows,

$\widetilde{M R}_{r j}=\tilde{A}_{r j}-\tilde{A}_{r, j-1}$.

The conventional moving range control chart introduced by Shewhart is used to construct fuzzy MR control chart. By using triangular fuzzy numbers, the fuzzy MR control chart is constituted as follows,

$\widetilde{U C L}_{M R_{r}}=D_{4} \overline{\widetilde{M R}}_{r}=D_{4}\left(\overline{M R}_{r L}, \overline{M R}_{r}, \overline{M R}_{r U}\right)$,

$\widetilde{C L}_{M R_{r}}=\overline{\widetilde{M R}}_{r}=\left(\overline{M R}_{r L}, \overline{M R}_{r}, \overline{M R}_{r U}\right)$,

$\widetilde{L C} L_{M R_{r}}=D_{3} \overline{\widetilde{M R}}_{r}=D_{3}\left(\overline{M R}_{r L}, \overline{M R}_{r}, \overline{M R}_{r U}\right)$,

where the values of $\mathrm{D}_{3}$ and $\mathrm{D}_{4}$ are function of the number of $\tilde{A}_{r j}$ 's used for calculating the related $\widetilde{M R}_{r j}$ and the table of their values appears in Montgomery(2005). We have only two $\tilde{A}_{r j}$ for calculating related $\widetilde{M R}_{r j}$, so the values of $\mathrm{D}_{3}$ and $\mathrm{D}_{4}$ always equal to 0 and 3.267 respectively. Center line, upper and lower control limits of fuzzy individuals control chart are obtained as follows,

$\widetilde{U C L}_{\tilde{A}_{r}}=\tilde{A}_{r}+3 \frac{\widetilde{\widetilde{M R}}_{r}}{d_{2}}=\left(A_{r L}, A_{r}, A_{r U}\right)+\frac{3}{d_{2}}\left(\overline{M R}_{r L}, \overline{M R}_{r}, \overline{M R}_{r U}\right)$,
$C L_{\tilde{A}_{r}}=\overline{\tilde{A}}_{r}=\left(\bar{A}_{r L}, \bar{A}_{r}, \bar{A}_{r U}\right)$,
$\widetilde{L C} L_{\tilde{A}_{r}}=\tilde{A}_{r}-3 \frac{\widetilde{M R}_{r}}{d_{2}}=\left(A_{r L}, A_{r}, A_{r U}\right)-\frac{3}{d_{2}}\left(\overline{M R}_{r L}, \overline{M R}_{r}, \overline{M R}_{r U}\right)$, 
where the values of $\mathrm{d}_{2}$ is function of the number of $\tilde{A}_{r j}$ 's used for calculating the related $\widetilde{M R}_{r j}$ and the table of their values appears in Montgomery(2005). We have only two $\tilde{A}_{r j}$ 's for calculating the related $\widetilde{M R}{ }_{r j}$ 's, so the value of $\mathrm{d}_{2}$ is always equal to 1.128 .

\section{Identification $\mathbf{j}^{\text {th }}$ Profile Status (In-Control/Out-of-Control)}

As aforementioned above, the control lines are fuzzy and consisted of an interval. Hence, to compare sample $j$ 's fuzzy regression coefficients with control chart limits and to facilitate the plotting of observations on the chart, we propose two methods. First method, named Direct Fuzzy Approach, is based on a technique for the comparison of fuzzy numbers without losing original information due to employing no transformative or representative techniques. The second method applies some fuzzy transformation techniques.

\subsection{Direct Fuzzy Approach (DFA)}

Based on fuzzy preference approach introduced by Chen(2001), we use the definition of the equation 11,12 and 13 to determine if the fuzzy observations are exhibited in-control. To specify the superiority of fuzzy triangular statistic $\tilde{A}_{r j}$ over fuzzy triangular $\widetilde{U C L}$, we compare the difference of two membership function with zero. Using the difference of two fuzzy numbers, $\tilde{A}_{r j}-\widetilde{U C L}$, one can specify the superiority in two numbers. Intuitively, $S_{1}$ indicates the $\alpha$-cut right hand side portion of $\tilde{A}_{r j}-\widetilde{U C L}$ membership function from $\mathrm{Y}$-axis and $S_{2}$ shows the $\alpha$-cut left hand side portion as shown in figure 4. We define $S, S_{1}, S_{2}, e_{1}$ and $e_{2}$ as follows,

$S_{1}=\int_{x>0} \mu_{\widetilde{A}_{r j}-\widetilde{U C L}}(x) d x, S_{2}=\int_{x<0} \mu_{\widetilde{A}_{r j}-\widetilde{U C L}}(x) d x$,

$S=S_{1}+S_{2} \quad S>0$

$e_{1}=\frac{s_{1}}{S}, \quad e_{2}=\frac{s_{2}}{S}$.

Obviously, we can see that $e 1+e 2=1$ according to the definition of them. Therefore, $e_{1}>0.5$ indicates the fuzzy value $\tilde{A}_{r j}$ is greater than the fuzzy value $\widetilde{U C L}$, consequently the related static give out an out-of-control signal. If $e_{1} \leq 0.5$, then the statistic $\tilde{A}_{r j}$ is equal to or smaller than $\widetilde{U C L}$, thus related static exhibits an in-control situation.

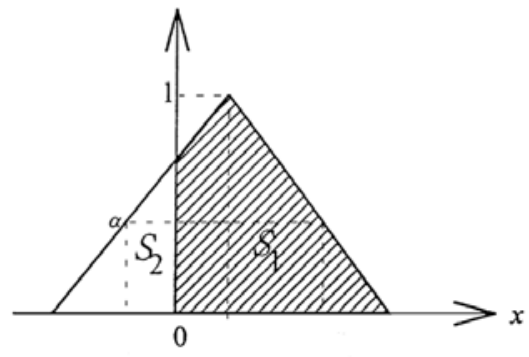

Fig. 4. An illustration of calculating $S_{1}$ and $S_{2}$

To specify the $\tilde{A}_{r j}$ beneath the fuzzy triangular $\widetilde{L C L}$, we apply the same approach. The value $\alpha$ in $\alpha$-cut measures the conservatism in determining the profile status. The greater $\alpha$ is, the more conservative it is. In practice, the value of $\alpha$ can be determined by the decision maker. Using this approach, the process is stable and in-control when four control charts display in-control condition for each sample.

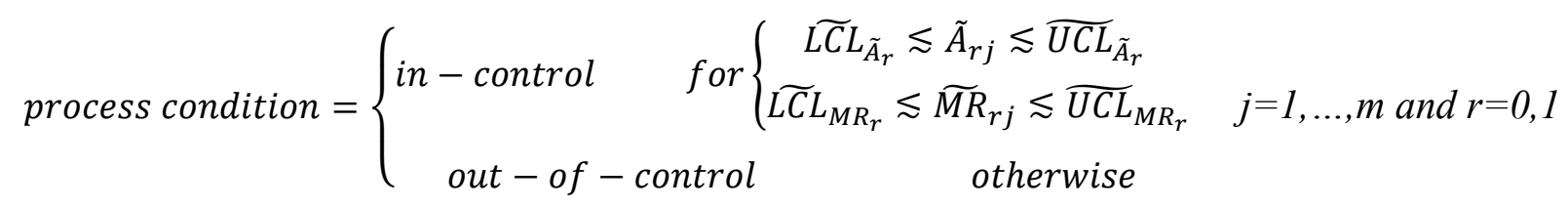




\subsection{Fuzzy Transformation Techniques}

To facilitate preserving the standard design of control charts and to ease the plotting of observations on the chart, it is necessary to convert the fuzzy values into scalars, which will be referred to as representative values, via some transformation techniques. There are four fuzzy transformation techniques: $\alpha$-level fuzzy midrange, $\alpha$-level fuzzy median, $\alpha$-level fuzzy average and $\alpha$-level fuzzy mode. Because the distribution of fuzzy data may be asymmetrical, the $\alpha$-level fuzzy midrange transformation technique is used for fuzzy individual \& moving range control charts.

$\alpha$-level fuzzy midrange is defined as the midpoint of the ends of the $\alpha$ level cut. It is not affected by the extreme values. Hence, it is a suitable measure for evaluating the central tendency of an asymmetrical distribution. $\alpha$-level fuzzy midrange transformation for fuzzy MR control chart is obtained as follows:

$$
\begin{aligned}
& U C L_{M R_{r}}=\frac{D_{4}\left(\overline{M R}_{r L}+\overline{M R}_{r U}\right)+\alpha D_{4}\left[\left(\overline{M R}_{r}-\overline{M R}_{r L}\right)-\left(\overline{M R}_{r U}-\overline{M R}_{r}\right)\right]}{2} \\
& C L_{M R_{r}}=\frac{\left(M R_{r j L}+M R_{r j U}\right)+\alpha\left[\left(M R_{r j}-M R_{r j L}\right)-\left(M R_{r j U}-M R_{r j}\right)\right]}{2} \\
& L C L_{M R_{r}}=\frac{D_{3}\left(\overline{M R}_{r L}+\overline{M R}_{r U}\right)+\alpha D_{3}\left[\left(\overline{M R_{r}}-\overline{M R}_{r L}\right)-\left(\overline{M R}_{r U}-\overline{M R}_{r}\right)\right]}{2}
\end{aligned}
$$

$\alpha$-level fuzzy midrange transformation for fuzzy individual control chart is created as follows,

$$
\begin{aligned}
U C L_{\tilde{A}_{r}} & =\frac{\left(A_{r L}+\frac{3}{d_{2}} \overline{M R}_{r L}+A_{r U}+\frac{3}{d_{2}} \overline{M R}_{r U}\right)+\alpha\left[\left(A_{r}+\frac{3}{d_{2}} \overline{M R}_{r}-A_{r L}-\frac{3}{d_{2}} \overline{M R}_{r L}\right)-\left(A_{r U}+\frac{3}{d_{2}} \overline{M R}_{r U}-A_{r}-\frac{3}{d_{2}} \overline{M R}_{r}\right)\right]}{2} \\
C L_{\tilde{A}_{r}} & =\frac{\left(A_{r L}+A_{r U}\right)+\alpha\left[\left(A_{r}-A_{r L}\right)-\left(A_{r U}-A_{r}\right)\right]}{2} \\
U C L_{\tilde{A}_{r}} & =\frac{\left(A_{r L}-\frac{3}{d_{2}} \overline{M R}_{r L}+A_{r U}-\frac{3}{d_{2}} \overline{M R}_{r U}\right)+\alpha\left[\left(A_{r}-\frac{3}{d_{2}} \overline{M R}_{r}-A_{r L}+\frac{3}{d_{2}} \overline{M R}_{r L}\right)-\left(A_{r U}-\frac{3}{d_{2}} \overline{M R}_{r U}-A_{r}+\frac{3}{d_{2}} \overline{M R}_{r}\right)\right]}{2}
\end{aligned}
$$

Then, the process is under control for each sample when four control charts exhibit in-control situation. It means that:

$\alpha$-Level Fuzzy Median transformation is defined as the point which partitions the curve under the membership function of a fuzzy set into equal regions. $\alpha$-level fuzzy median transformation of fuzzy moving range control chart is obtained as follows,

$$
\begin{aligned}
& U C L_{M R_{r}}=\frac{D_{4}\left(\overline{M R}_{r L}+\overline{M R}_{r}+\overline{M R}_{r U}\right)+\alpha D_{4}\left[\left(\overline{M R}_{r}-\overline{M R}_{r L}\right)-\left(\overline{M R}_{r U}-\overline{M R}_{r}\right)\right]}{3} \\
& C L_{M R_{r}}=\frac{\left(\overline{M R}_{r L}+\overline{M R}_{r}+\overline{M R}_{r U}\right)+\alpha\left[\left(\overline{M R}_{r}-\overline{M R}_{r L}\right)-\left(\overline{M R}_{r U}-\overline{M R}_{r}\right)\right]}{3} \\
& L C L_{M R_{r}}=\frac{D_{3}\left(\overline{M R}_{r L}+\overline{M R}_{r}+\overline{M R}_{r U}\right)+\alpha D_{3}\left[\left(\overline{M R}_{r}-\overline{M R}_{r L}\right)-\left(\overline{M R}_{r U}-\overline{M R}_{r}\right)\right]}{3}
\end{aligned}
$$

$\alpha$-level fuzzy median transformation for fuzzy individual control chart is created as follows:

$$
\begin{aligned}
U C L_{\tilde{A}_{r}}= & \left(A_{r L}+\frac{3}{d_{2}} \overline{M R}_{r L}+A_{r}+\frac{3}{d_{2}} \overline{M R}_{r}+A_{r U}+\frac{3}{d_{2}} \overline{M R}_{r U}\right)+\alpha\left[\left(A_{r}+\frac{3}{d_{2}} \overline{M R}_{r}-A_{r L}-\frac{3}{d_{2}} \overline{M R}_{r L}\right)-\left(A_{r U}+\frac{3}{d_{2}} \overline{M R}_{r U}-A_{r}-\frac{3}{d_{2}} \overline{M R}_{r}\right)\right] \\
C L_{\tilde{A}_{r}} & =\frac{\left(A_{r L}+A_{r}+A_{r U}\right)+\alpha\left[\left(A_{r}-A_{r L}\right)-\left(A_{r U}-A_{r}\right)\right]}{3} \\
L C L_{\tilde{A}_{r}} & =\frac{\left(A_{r L}-\frac{3}{d_{2}} \overline{M R}_{r L}+A_{r}-\frac{3}{d_{2}} \overline{M R}_{r}+A_{r U}-\frac{3}{d_{2}} \overline{M R}_{r U}\right)+\alpha\left[\left(A_{r}-\frac{3}{d_{2}} \overline{M R}_{r}-A_{r L}+\frac{3}{d_{2}} \overline{M R}_{r L}\right)-\left(A_{r U}-\frac{3}{d_{2}} \overline{M R}_{r U}-A_{r}+\frac{3}{d_{2}} \overline{M R}_{r}\right)\right]}{3}
\end{aligned}
$$

Then, a signal is produced whenever one of the four charts produces an out-of-control signal. In other words, the process is under control when four control charts exhibit in-control situation for each sample.

If all control charts state in-control situation, the process is stable; the regression parameters can be estimated to use in phase II online monitoring of fuzzy profiles. One should use Eq. (18) to monitor the 
profiles through some univariate control charts. Alternatively, Eq. (19) can be utilized if multivariate control chart are going to be used in phase II.

$\tilde{A}_{r}=\frac{\sum_{j=1}^{m} \tilde{A}_{r j}}{m} \quad r=0,1$

$\tilde{B}_{r}=\frac{\sum_{j=1}^{m} \tilde{B}_{r j}}{m} \quad r=0,1$

\section{Case Study}

Customer satisfaction is a criterion for company performances in meeting customer needs. In this application, we consider a tour where the customer satisfaction index is measuring and monitoring by using the questionnaire. Customer satisfaction index makes quality profile in survey duration which consists of fuzzy quality response. By using our developed approach, tourist satisfaction is monitored and barriers to achieving the desired level of customer satisfaction are identified and removed. Quality characteristic in this case study is tourist satisfaction known as response variable in tour profile which has been measured for each tourist at the end of journey day. Descriptive variable is the day of journey also known as independent variable in tour profile. Tourist satisfaction is a fuzzy QC, thus it is measured in the basis of some linguistic terms as shown in Fig. 5. Two hundred tourists' satisfaction is measured during 7-day trip in this study.

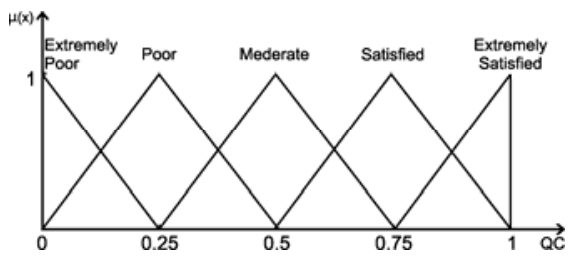

Fig. 5. Membership function of each linguistic term for tourist satisfaction

A regression profile is established for each tourist by using linear programming in Eq. (6). The intercept $A_{0}$ and the slope $A_{1}$ are fuzzy triangular numbers as shown in Table $1 . h$-cut, $w_{1}$ and $w_{2}$ are model parameters and were determined subject to the highest similarity between observed and predicted values in each of the profiles have been obtained.

Table 1

200 tourists' profile (h-cut $=0.25, \mathrm{w}_{1}=0.4, \mathrm{w}_{2}=0.6$ )

\begin{tabular}{|c|c|c|c|c|}
\hline \multirow[b]{2}{*}{ Sample j } & \multicolumn{4}{|c|}{ Fuzzy Profile coefficient } \\
\hline & Center of $A_{0}$ & Center of $A_{1}$ & Spread of $A_{0}$ & Spread of $A_{1}$ \\
\hline 1 & 0.668904 & 0.049199 & $9.78 \mathrm{E}-19$ & $3.47 \mathrm{E}-19$ \\
\hline 2 & 0.9000 & $-1.5 \mathrm{E}-10$ & $2.57 \mathrm{E}-12$ & $4.93 \mathrm{E}-13$ \\
\hline 3 & 0.5500 & 0.1000 & $2.09 \mathrm{E}-17$ & $2.52 \mathrm{E}-18$ \\
\hline 4 & 0.6750 & 0.0750 & $6.5 \mathrm{E}-18$ & $1.42 \mathrm{E}-19$ \\
\hline “ & “ & “ & " & “ \\
\hline 197 & 0.6500 & 0.1250 & $1.95 \mathrm{E}-15$ & $2.15 \mathrm{E}-16$ \\
\hline 198 & 0.735308 & 0.093243 & 1.69E-14 & $3.9 \mathrm{E}-15$ \\
\hline 199 & 0.6500 & -0.08333 & $1.4 \mathrm{E}-13$ & $3.02 \mathrm{E}-14$ \\
\hline 200 & 0.5500 & 0.0500 & $7.82 \mathrm{E}-16$ & 7.19E-17 \\
\hline
\end{tabular}

Fuzzy I-MR control chart should be utilized for monitoring both slope and intercept of each profile to identify any out-of-control situation. Here, we try to identify any out-of-control regression lines and then to remove them from the data set in order to estimate strictly the in-control regression parameters for future use in phase II online monitoring. 


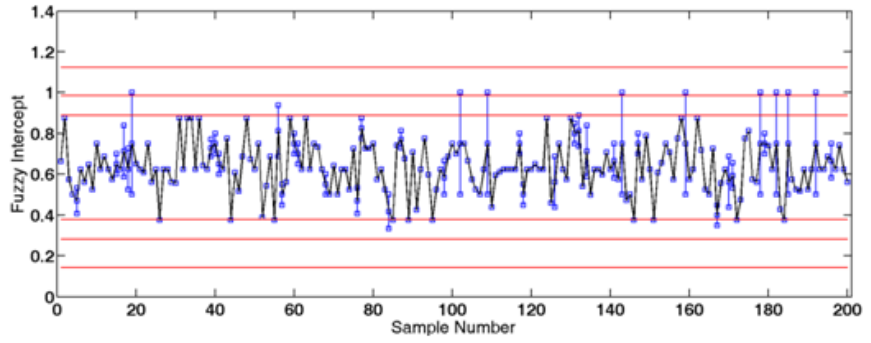

Fig. 6. Fuzzy M-R control chart for intercept $A_{0}$

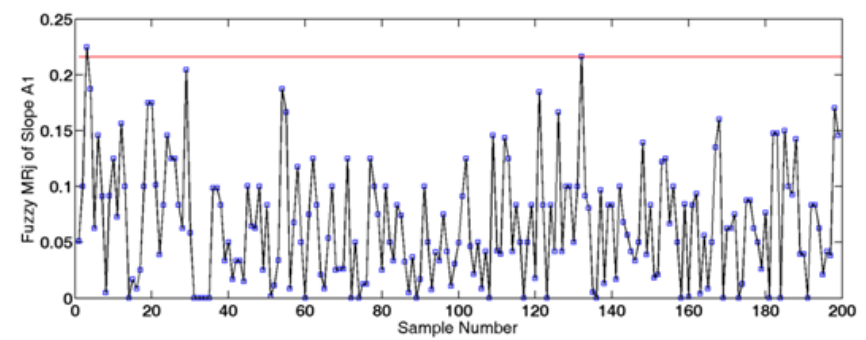

Fig. 8. Fuzzy M-R control chart for intercept $A_{1}$

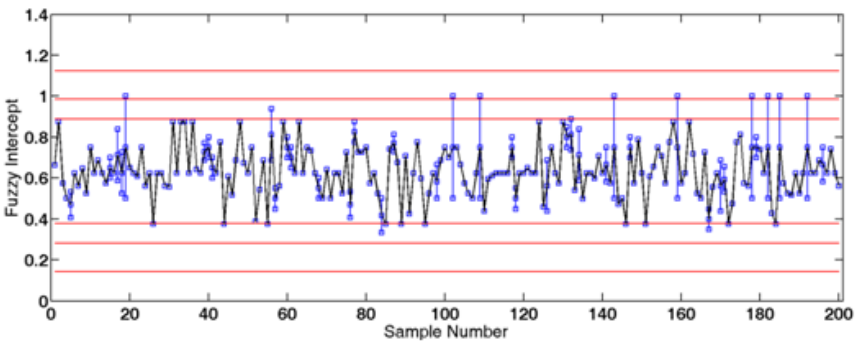

Fig. 7. Fuzzy individual control chart for intercept $\mathrm{A}_{0}$

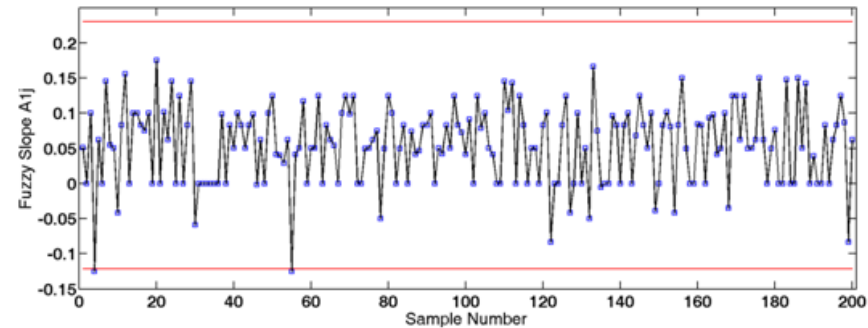

Fig. 9. Fuzzy individual control chart for slope $A_{1}$

Each profile statistic is calculated and are plotted on the control charts as illustrated in Figure 6 to 9. If the computed statistic is within the related control chart limites, the process is in control, otherwise we face to an out-of-control process. It can be seen that 22 points state out-of-control signals in The I-MR control charts. It means that the satisfaction of 22 tourist are not in natural conditions. Thus, samples related to these out-of-control statistics should be eliminate to construct the new modified control limit.

The in-control process is achieved after 4 loops of chart sketching by using 168 tourists' data and the new control limit is obtained. Therefore the resulting control chart with thresholds shown in table 2 will be used for online monitoring of process in Phase II.

\section{Table 2}

Control limits of fuzzy I-MR chart

\begin{tabular}{cccccccc}
\hline \multirow{2}{*}{$\begin{array}{c}\text { Profile } \\
\text { Parameter }\end{array}$} & Control & \multicolumn{2}{c}{ Fuzzy M-R control chart } & \multicolumn{2}{c}{ Fuzzy Individuals control chart } \\
\cline { 2 - 7 } & Limits & Left & Center & Right & Left & Center & Right \\
\hline \multirow{2}{*}{$\mathbf{A}_{\mathbf{0}}$} & UCL & 0.3398 & $\mathbf{0 . 4 3 2 7}$ & 0.5757 & 0.8888 & $\mathbf{0 . 9 8 6 2}$ & 1.1243 \\
& CL & 0.104 & $\mathbf{0 . 1 3 2 4}$ & 0.1762 & 0.6122 & $\mathbf{0 . 6 3 3 9}$ & 0.6557 \\
& LCL & 0.0000 & $\mathbf{0 . 0 0 0 0}$ & 0.0000 & 0.1435 & $\mathbf{0 . 2 8 1 7}$ & 0.3791 \\
\hline \multirow{3}{*}{$\mathbf{A}_{\mathbf{1}}$} & UCL & 0.2162 & $\mathbf{0 . 2 1 6 2}$ & 0.2162 & 0.2303 & $\mathbf{0 . 2 3 0 3}$ & 0.2303 \\
& CL & 0.0662 & $\mathbf{0 . 0 6 6 2}$ & 0.0662 & 0.0543 & $\mathbf{0 . 0 5 4 3}$ & 0.0543 \\
& LCL & 0.0000 & $\mathbf{0 . 0 0 0 0}$ & 0.0000 & -0.1217 & $\mathbf{- 0 . 1 2 1 7}$ & -0.1217 \\
\hline
\end{tabular}

\section{Discussion}

Model parameters for constructing fuzzy profiles are $h$-cut, $w_{1}$ and $w_{2}$ in linear programming. Parameters changes have some impact on the quality of profile constructing. Mis-selection the model parameters lead to misunderstanding about the process situation. The spread of estimated fuzzy response value should be narrow enough to be of use and should be wide enough to contain as many observations as possible. Hence, to determine appropriate values for the parameters of the model, we use the similarity index developed by Sridevi \& Nadarajan(2009) in order to measure the similarity of estimated and observed values to investigate the impacts of parameter changes on profile construction.

According to DOE approach, we initially determine 4 levels for $h$-cut and 11 levels for $w_{1}$ and $w_{2}$ as shown in Table 3. 
Table 3

Similarity index for different run

\begin{tabular}{|c|c|c|c|c|c|c|c|c|c|c|c|}
\hline h-cut & & & & & & $\mathbf{0}$ & & & & & \\
\hline $\mathrm{w}_{1}$ & 0 & 0.1 & 0.2 & 0.3 & 0.4 & 0.5 & 0.6 & 0.7 & 0.8 & 0.9 & 1 \\
\hline $\mathrm{w}_{2}$ & 1 & 0.9 & 0.8 & 0.7 & 0.6 & 0.5 & 0.4 & 0.3 & 0.2 & 0.1 & 0 \\
\hline similarity & 0.8532 & 0.8309 & 0.8309 & 0.8494 & 0.8657 & 0.8734 & 0.8527 & 0.8446 & 0.8445 & 0.8448 & 0.8518 \\
\hline h-cut & & & & & & 0.25 & & & & & \\
\hline $\mathrm{w}_{1}$ & 0 & 0.1 & 0.2 & 0.3 & 0.4 & 0.5 & 0.6 & 0.7 & 0.8 & 0.9 & 1 \\
\hline $\mathrm{w}_{2}$ & 1 & 0.9 & 0.8 & 0.7 & 0.6 & 0.5 & 0.4 & 0.3 & 0.2 & 0.1 & 0 \\
\hline similarity & 0.8535 & 0.8311 & 0.8434 & 0.8569 & 0.8755 & 0.8666 & 0.8503 & 0.8504 & 0.8503 & 0.8503 & 0.8530 \\
\hline h-cut & & & & & & 0.5 & & & & & \\
\hline $\mathrm{w}_{1}$ & 0 & 0.1 & 0.2 & 0.3 & 0.4 & 0.5 & 0.6 & 0.7 & 0.8 & 0.9 & 1 \\
\hline $\mathrm{w}_{2}$ & 1 & 0.9 & 0.8 & 0.7 & 0.6 & 0.5 & 0.4 & 0.3 & 0.2 & 0.1 & 0 \\
\hline similarity & 0.8556 & 0.8312 & 0.8516 & 0.8730 & 0.8689 & 0.8588 & 0.8587 & 0.8588 & 0.8587 & 0.8586 & 0.8539 \\
\hline h-cut & & & & & & 0.75 & & & & & \\
\hline $\mathrm{w}_{1}$ & 0 & 0.1 & 0.2 & 0.3 & 0.4 & 0.5 & 0.6 & 0.7 & 0.8 & 0.9 & 1 \\
\hline $\mathrm{w}_{2}$ & 1 & 0.9 & 0.8 & 0.7 & 0.6 & 0.5 & 0.4 & 0.3 & 0.2 & 0.1 & 0 \\
\hline similarity & 0.8564 & 0.8511 & 0.8753 & 0.8688 & 0.8686 & 0.8686 & 0.8686 & 0.8685 & 0.8685 & 0.8685 & 0.8545 \\
\hline
\end{tabular}

Then the similarity index is calculated for 7 pairs of fuzzy estimated and observed values in 200 profiles for each run. The maximum similarity are around the coordinate of $h$-cut $=0.25, w_{1}=0.4$ and $w_{2}=0.6$ as shown in Fig. 10. Hence, We use this coordinate for other operations in fuzzy profile monitoring.

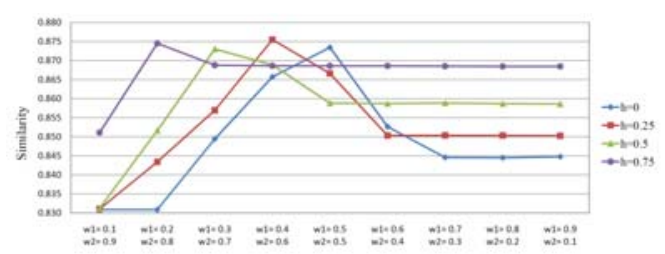

Fig. 10. Similarity of observed and estimated values

In this section by using an instant satisfaction data in table 4, we investigate decision maker's preferences in two-objective linear programming which is showed by weight factors $w_{1} \& w_{2}$. The factor $w_{1}$ shows the importance of enveloping the $h$-cut of the observed values by the $h$-cut of the estimated values and $w_{2}$ illustrates the importance of minimizing the difference between predicted and observed values.

\section{Table 4}

Sample tourist satisfaction

\begin{tabular}{cccccccc}
\hline Tourist & Day 1 & Day 2 & Day 3 & Day 4 & Day 5 & Day 6 & Day 7 \\
\hline 98 & poor & poor & moderate & moderate & satisfied & moderate & satisfied \\
\hline
\end{tabular}

As shown in Fig. 11, the changes in weight factor $\mathrm{w}_{1}$ has the main affect on the with regression line so that the expanse of fuzzy regression line decreases while increasing the $w_{1}$ value and the resulted regression line leads to producing non-fuzzy values. The weight factor $W_{2}$ influences estimation accuracy. We expect to increase the estimate error while $W_{2}$ is being reduced, but because we are dealing with fuzzy data, the width of the regression line is another effective factor in reducing forecast error. Therefore, the intermediate values will develop the best result according to the Fig. 10. The changes of $h$-cut values influence fuzzy regression lines according to figure 12 . $h$-cut determines the interval of observed and predicted values used in linear programming to develop a regression equation. Sample Regression line in Fig. 12 has been obtained through different values of $h$-cut with constant values for $w_{1}$ and $w_{2}$ which are equal to 0.4 and 0.6 , respectively. 

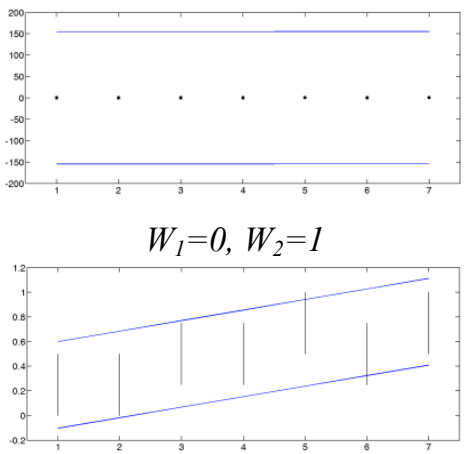

$W_{1}=0.3, W_{2}=0.7$

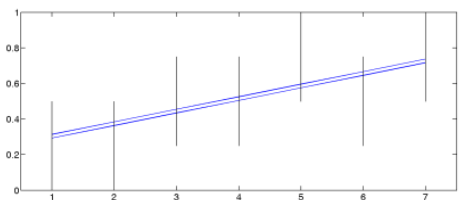

$W_{1}=0.6, W_{2}=0.4$

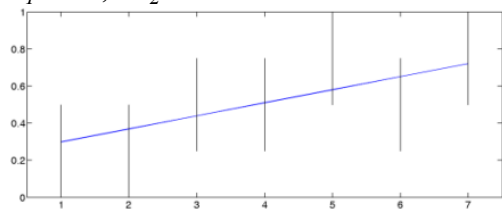

$W_{1}=0.9, W_{2}=0.1$

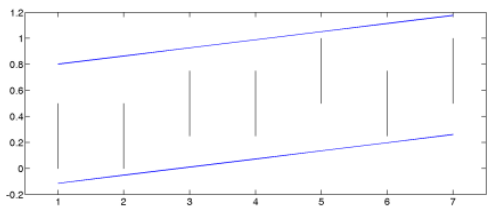

$W_{1}=0.1, W_{2}=0.9$

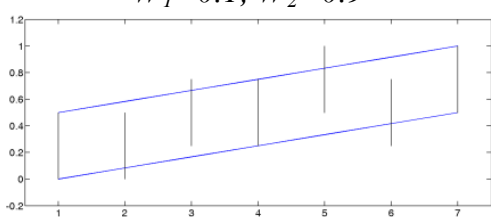

$W_{1}=0.4, W_{2}=0.6$

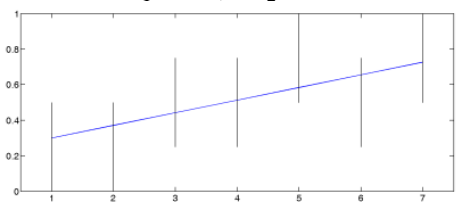

$W_{1}=0.7, W_{2}=0.3$

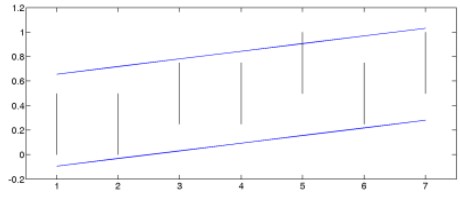

$W_{1}=0.2, W_{2}=0.8$

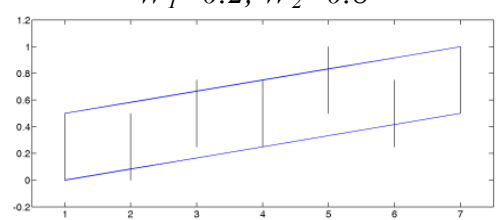

$W_{1}=0.5, W_{2}=0.5$

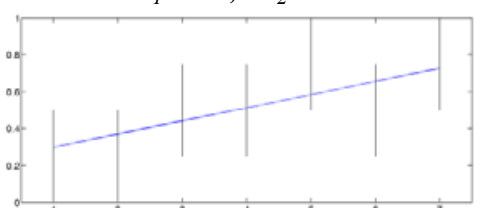

$W_{1}=0.8, W_{2}=0.2$

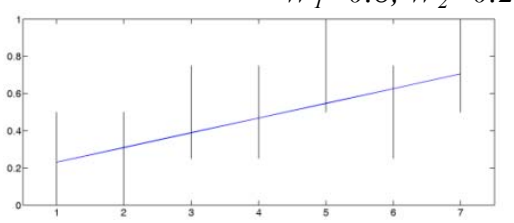

$W_{1}=1, W_{2}=0$

Fig. 11. Fuzzy Profile for sample \#5 at h-cut 0.25

It can be seen that the width of regression lines is reduced while h-cut increases. If the $h$-cut becomes greater, the specified interval becomes smaller and converges to a point. Therefore, fuzzy regression line yielded is converted to a conventional crisp regression line as a result of increasing $h$-cut. If the decision maker is risk averter, he will prefer the $h$-cut of the predicted values to have a broad width.

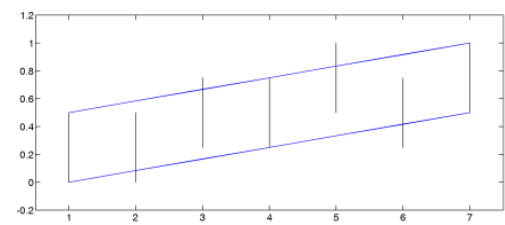

$h-c u t=0$

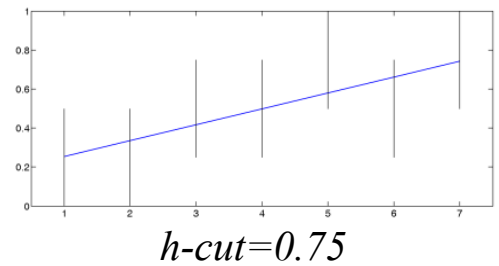

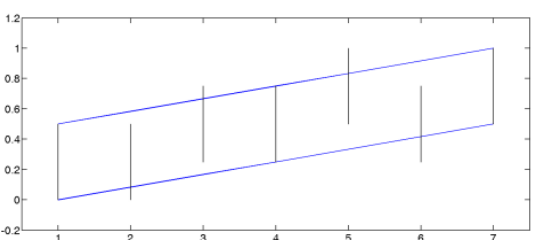

$h-c u t=0.25$

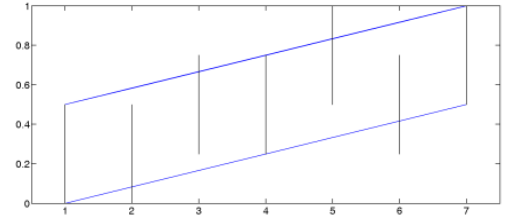

$h-c u t=0.5$

Fig. 12. Fuzzy Profile via different values of $h$-cut at $w_{1}=0.4 \& w_{2}=0.6$

\section{Conclusion}

In this paper, we developed a framework for monitoring process/product profiles for the first time in fuzzy environment where the response quality characteristic is fuzzy. A model in fuzzy linear regression has been developed to construct the quality profiles by using linear programming approach. The developed model for constructing fuzzy profiles has some important advantages. It considers two objectives in modeling fuzzy linear regression mutually. It decreases the width of the regression coefficients and minimizes the difference between the observed and the predicted values. In addition, Fuzzy individuals and moving-range (I-MR) control charts were developed to check the stability of the fuzzy functional relationship and to estimate the in-control process parameters. A case study about 
customer satisfaction was presented to illustrate the application of our approach and demonstrate the sensitivity analysis of parameters in constructing a fuzzy profile.

The stages of our approach for monitoring fuzzy Profiles in phase-I are summarized as follows,

1. Gather some historical data sample by sample (number of samples should not be less than $\mathrm{m}$ (generally $\mathrm{m}=30$ ).

2. Construct fuzzy linear regression for each sample using developed linear programming model.

3. Construct two fuzzy moving range control charts for $\tilde{A}_{0}$ and $\tilde{A}_{1}$, Construct two fuzzy individual control charts for $\tilde{A}_{0}$ and $\tilde{A}_{1}$. (These four charts are used for diagnostic purposes).

4. Check whether moving range of each sample's intercept and slope are within the related control limits.

5. Check whether each sample intercept and slope are within the related fuzzy individual control limits.

6. If all the control charts state in-control situation go to step 10 otherwise, go to step 8 .

7. Identify the corresponding out-of-control samples, discover assignable causes, and remove the tainted samples from the data set.

8. If the number of samples is not less than $\mathrm{m}$, go to step 4, otherwise go to step 1 .

9. The process is stable; the regression parameters can be estimated for use in phase II online monitoring of fuzzy profiles. One should use the Eq. (18) to monitor the profiles through some univariate control charts. Eq. (19) should be utilized if multivariate control chart are going to be used for phase II.

\section{References}

Amiri, A., Eyvazian, M., Zou, C. \& Noorossana, R. (2012). A parameters reduction method for monitoring multiple linear regression profiles. International Journal of Advanced Manufacturing Technology, 58(5-8), 621-629.

Azadeh, A., Saberi, M. \& Seraj, O. (2010). An integrated fuzzy regression algorithm for energy consumption estimation with non-stationary data: A case study of Iran. Energy , 35, 2351-2366.

Chang, Y. \& Ayyub, B. M. (2001). Fuzzy regression methods- a comparative assessment. Fuzzy Sets and Systems, 119, 187-203.

Chen, C.T. (2001). A fuzzy approach to select the location of the distribution center. Fuzzy Sets and Systems, 118, 65-73.

Chen, S. \& Nembhard, H.B. (2011). High-dimensional control chart for profile monitoring. Quality and Reliability Engineering International, 27(4), 451-464.

Croarkin, C. \& Varner, R. (1982). Measurement assurance for dimensional measurements on integrated-circuit photomasks. NBS Technical Note 1164. US Department of Commerce, Washington, D.C.

Erginel, N. (2008). Fuzzy individual and moving range control charts with $\alpha$-cuts. Journal of Intelligent \& Fuzzy Systems, 19, 373-383.

Eyvazian, M., Noorossana, R., Saghaei, A. \& Amiri, A. (2011). Phase II monitoring of multivariate multiple linear regression profiles. Quality and Reliability Engineering International, 27(3), 281296.

Gupta, S., Montgomery, D. C. \& Woodall, W. H. (2006). Performance Evaluation of Two Methods for Online Monitoring of Linear Calibration Profiles. International journal of Production Research, 44, 1927-1942.

Hojati, M., Bector, C.R. \& Smimou, K. (2005), A Simple method for computation of fuzzy linear regression. European Journal Of Operational Research, 166(1), 172-184.

Hosseinifard, S.Z., Abdollahian, M. \& Zeephongsekul, P. (2011). Application of artificial neural networks in linear profile monitoring. Expert Systems with Applications, 38(5), 4920-4928.

Jensen, W. A., Birch, J. B. \& Woodall, W. H. (2008). Monitoring Correlation Within Linear Profiles Using Mixed Models. Journal of Quality Technology, 40, 167-183. 
Kang, L. \& Albin, S. L. (2000). On-Line Monitoring When the Process Yields a Linear Profile. Journal of Quality Technology, 32, 418-426.

Kim, K., Mahmoud, M.A. \& Woodall, W. H. (2003). On the monitoring of linear profiles. Journal of Quality Technology, 35, 317-328.

Mahmoud, M.A., Parker, P.A., Woodall, W.H. \& Hawkins, D.M. (2007). A change point method for linear profile data. Quality and Reliability Engineering International, 23(2), 247-268.

Mahmoud, M. A. \& Woodall, W. H. (2004). Phase I monitoring of linear profiles with calibration application. Technometrics, 46, 380-391.

Montgomery, D.C. (2005). Introduction to statistical quality control. Wiley, New York.

Noorossana, R., Eyvazian, M., Amiri, A. \& Mahmoud M. A. (2010). Statistical Monitoring of Multivariate Multiple Linear Regression Profiles in Phase I with Calibration Application. Quality and Reliability Engineering International, 26(3), 291-303.

Noorossana, R., Eyvazian, M. \& Vaghefi, S. A. (2010). Phase II monitoring of multivariate simple linear profiles. Computers and Industrial Engineering, 58, 563-570.

Noorossana, R., Vaghefi, A. \& Dorri, M. (2011). Effect of non-normality on the monitoring of simple linear profiles. Quality and Reliability Engineering International, 27(4), 425-436.

Noorossana, R., Amiri, A., Vaghefi, S. A. \& Roghanian, E. (2004). Monitoring Quality Characteristics

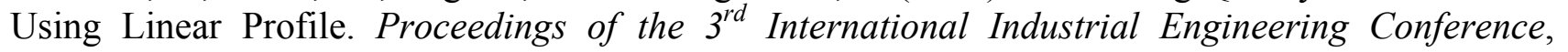
Tehran, Iran, 246-255.

Noorossana, R. Amiri \& A. Soleimani, P. (2008). On the Monitoring of Auto-correlated Linear Profiles. Communications in Statistics, Theory and Methods, 37, 425-442.

Noorossana, R., Eyvazian, M. \& Vaghefi, A. (2011). Phase II monitoring of multivariate simple linear profiles. Quality and Reliability Engineering International, 27(3), 281-296

Noorossana, R., Eyvazian, M., Amiri, A. \& Mahmoud, M.A. (2010). Statistical monitoring of multivariate multiple linear regression profiles in phase I with calibration application. Quality and Reliability Engineering International, 26(3), 291-303.

Ozelkan, E.C. \& Duckstein, L. (2000), Multi-objective fuzzy regression: A general framework. Computers and Operation Research, 27, 635-652.

Saghaei, A., Mehrjoo M. \& Amiri A. (2009). A CUSUM-based method for monitoring simple linear profiles. International Journal of Advanced Manufacturing Technology, 45,1252-1260.

Savic, D.A. \& Pedrycz, W. (1991). Evaluation of fuzzy linear regression models. Fuzzy Sets and Systems, 39, 51-63.

Sridevi, B. \& Nadarajan, R. (2009). Fuzzy Similarity Measure for Generalized Fuzzy Numbers. International Journal of Open Problems in Computer Science and Mathematics, 2(2), 240-253.

Tanaka, H., Uejima, S. \& Asai, K. (1982). Linear regression analysis with fuzzy model. IEEE Transactions Systems, Man, and Cybernetics, 12, 903-907.

Walker, E. \& Wright, S. (2002). Comparing Curves Using Additive Models. Journal of Quality Technology, 34, 118-129.

Wang, H.F. \& Tsaur, R.C. (2000 a). Insight of a fuzzy regression model. Fuzzy Sets and Systems, 112, 355-69.

Wang, H.F. \& Tsuar, R.C. (2000 b). Resolution of fuzzy regression model. European Journal of Operational Research, 126, 637-50.

Zhang, J., Li, Z. \& Wang, Z. (2009). Control Chart Based on Likelihood Ratio for Monitoring Linear Profiles. Computational Statistics and Data Analysis, 53, 1440-1448.

Zhu, J. \& Lin, D.K.J. (2010). Monitoring the Slopes of Linear Profiles. Quality Engineering, 22(1), 112

Zou, C., Zhang, Y. \& Wang, Z. (2006). Control Chart Based on Change-Point Model for Monitoring Linear Profiles. IIE Transactions, 38, 1093-1103.

Zou, C., Ning, X. \& Tsung, F. (2012). LASSO-based multivariate linear profile monitoring. Annals of Operations Research, 192(1), 3-19. 